Paper

\title{
Neural-based routing strategy with transmission information for complex communication networks
}

\author{
Takayuki Kimura ${ }^{1 a)}$, Tohru Takamizawa ${ }^{2 b)}$, Keisuke Kimura $^{2 c)}$, \\ and Kenya Jin'no ${ }^{1 \text { d) }}$ \\ ${ }^{1}$ Department of Electrical and Electronic Engineering, \\ Nippon Institute of Technology, \\ 4-1-1 Gakuendai, Miyasiro, Minami-Saitama 345-8501, Japan \\ ${ }^{2}$ Graduate School of Electronics, Information and Media Engineering, \\ Nippon Institute of Technology, \\ 4-1-1 Gakuendai, Miyasiro, Minami-Saitama 345-8501, Japan \\ a) tkimura@nit.ac.jp \\ b)e1102304@estu.nit.ac.jp \\ c)e1092204@estu.nit.ac.jp \\ d) jinno@nit.ac.jp
}

Received September 3, 2014; Revised November 15, 2014; Published April 1, 2015

\begin{abstract}
Because of the huge growth in the number of Internet users, data packets flowing in communication networks have also growth, and as a result, some packets can become congested in communication networks. If packet congestion occurs in a communication network, the packets are trapped in congested nodes, and then the transmission of these packets to their destinations is delayed. Further, the packets could be removed from the communication network in the worst case. To overcome these undesirable problems, an efficient routing strategy based on mutually connected neural networks has been proposed. This neural-based routing strategy shows good performance for regular topological communication networks. However, the performance of the routing strategy declines in irregular topological communication networks. To improve its performance for irregular topological communication networks, we propose in this paper a new neural-based routing strategy with the transmission information. Numerical experiments show that the performance of the proposed strategy is enhanced by the newly added transmission information as compared to the conventional routing strategies. Further, the proposed routing strategy shows better performance for other topological complex communication networks.
\end{abstract}

Key Words: mutually connected neural network, decentralized control, packet communication network, complex network, congestion control 


\section{Introduction}

Recently, the number of users of packet communication networks such as the Internet has drastically increases because of the widespread use of mobile devices. In such communication networks, data flow is divided into small portions of data, namely, packets. Exchanging data in a packet communication network is achieved by transmitting and receiving these data packets. If the number of packets in flow increases enough, packet congestion can occur [1]. Packets then become trapped in the congested nodes and their transmission to the destinations is delayed. Further, the packets could be removed from the communication network in the worst case. To avoid such undesirable situations, packet routing is used to control packet flow in packet communication networks.

There are several shortest path approaches for packet routing in real-world systems; for example, Dijkstra's algorithm [2], Bellman-Ford strategy [3], Extended Dijkstra's algorithm [4], and WarshallFloyd algorithm [5]. However, it has been shown that the shortest path protocol commonly employed by communication networks faces serious challenges if the data volume continues to increase [6]. Specifically, the shortest path protocol transmits data using only the distance information of the communication network, and the routers through which a large number of shortest paths go easily become congested. Thus, it is imperative to enhance the transmission algorithm to ensure reliable communication through the network. Further, an understanding of the data flow dynamics of the packets is necessary. For example, Ohira et al. [1] studied the optimal network structure for packet flow in communication network and showed the onset of phase transition behavior from a free-flow state to congested state as the packet creation rate increases. Arenas et al. [7] analyzed this phase transition behavior for hierarchical branching networks. Zhao et al. [8] studied the dynamics of flowing packets for various network topologies, and showed that networks that are significantly heterogeneous property inhibit packet congestion.

There are two strategies for routing packets. The first one is a centralized control strategy that determines all of the routes for the packets using a centralized control unit. This routing strategy exhibits good performance for small communication networks. However, if the size of the communication network becomes large, the computational load of the central control unit increases, and then it is impossible to control the transmissions of all the packets to their destinations using the central control unit. The second strategy is a decentralized control strategy. Each node autonomously determines the optimal adjacent nodes for routing packets in this case. A decentralized control strategy is more suitable for large communication networks.

One efficient decentralized control strategy is a routing strategy based on a mutually connected neural network proposed by Horiguchi and Ishioka [9]. This strategy searches for the optimum path of a packet to its destination using the energy minimization principle of a mutually connected neural network. It is well known that a mutually connected neural network is an effective way to solve hard classes of problems, namely, $\mathscr{N} \mathscr{P}$-hard problems. This routing strategy [9] shows good performance when it is applied to regular topological communication networks. However, its performance declines for irregular topological communication networks because of the nonuniform distribution of packets [10]. To improve its performance in this case, we propose in this paper a new neural-based routing strategy with transmission information. In addition, we evaluate the proposed neural-based routing strategy using irregular topological communication networks [10]. Further, we compare the performance of the proposed strategy with the conventional strategy with various types of complex communication networks.

This paper is organized as follows. In Section 2, we briefly describe the routing strategy based on mutually connected neural networks and clarify the reason for its performance decline in the case of irregular topological communication networks. In Section 3, we propose a neural-based routing strategy with two types of the transmission information and evaluate the effectiveness of these proposed routing strategies in Section 4. Finally, we conclude the paper in Section 5.

\section{Routing strategy based on mutually connected neural networks}

Horiguchi and Ishioka proposed a method for solving the packet routing problem using a mutually 
connected neural network [9]. Our proposed routing strategy in this paper is based on this routing strategy. We first briefly describe the routing strategy using a mutually connected neural network [9] in this section. A model of the communication network consists of nodes and links. Each packet is transmitted to the nodes according to the FIFO (First-In First-Out) principle. Each node receives multiple packets simultaneously and has a buffer to store some of the packets. The packets are removed from the communication network if the packets are transmitted to nodes that have full buffers. In addition, the packets are also removed when they exceed a fixed threshold of packet movements.

The communication network model has $N$ nodes, where the $i$ th node is connected to $N_{i}$ adjacent nodes. Hence, a mutually connected neural network that has $N_{i}$ neurons is assigned to each node.



Fig. 1. Schematic representation of the neural network in this paper. In this example, the $i$ th node has three adjacent nodes. The neural network of the $i$ th node is therefore constructed by three neurons that correspond to adjacent nodes 1,2 , and $l$.

Figure 1 shows an example of the communication network model. In the mutually connected neural network, the $i$ th neuron corresponds to the connection between the $i$ th and $l$ th nodes. The routing strategy based on this mutually connected neural network determines the transmission node by minimizing an energy function defined as follows:

$$
\begin{aligned}
E= & \frac{1}{2} \sum_{i=1}^{N} \sum_{k=1}^{N_{i}} \sum_{l=1}^{N_{i}} J_{i k, i l} v_{i k} v_{i l}-\eta \sum_{i=1}^{N} \sum_{l=1}^{N_{i}}\left\{1-\frac{1}{b_{l}}\left(q_{l}+\frac{1}{2} \sum_{j \neq i}^{N_{l}} v_{j l}\right)\right\} v_{i l} \\
& -(1-\eta) \sum_{i=1}^{N} \sum_{l=1}^{N_{i}}\left(1-\frac{d_{l}}{d_{c}}\right) v_{i l}+\xi \sum_{i=1}^{N}\left(\sum_{l=1}^{N_{i}} v_{i l}-1\right)^{2},
\end{aligned}
$$

where $J_{i k, i l}$ is the connection weight between the $i k$ th neuron and the $i l$ th neuron, $b_{l}$ is the buffer size of the $l$ th adjacent node, $q_{l}$ is the number of stored packets in the $l$ th adjacent node, $d_{l}$ is the shortest distance from the $l$ th adjacent node to the destination of the packet at the $i$ th node, $d_{c}$ is the longest distance among any shortest paths, $\eta$ is the control parameter that decides the priorities of the first and second terms, and $\xi$ is the control parameter that guarantees the uniqueness of the firing neurons.

In Eq. (1), the second term expresses the load distribution of the adjacent nodes, and the third term expresses the distance of the packet from the $i$ th node to the destination. The last term guarantees that only a single neuron fires in each neural network. By minimizing this energy function, the $l$ th adjacent node is easily selected as the transmitted node from the $i$ th node if the distance to the destination is the shortest from the $l$ th adjacent node and the $l$ th adjacent node has a small number of packets in its buffer.

The internal state of the mutually connected neural network is updated using the same method as the one proposed by Hopfield and Tank [11]. The output of the $i$ th neuron $v_{i l}$ is described as follows: 


$$
v_{i l}= \begin{cases}\frac{1}{1+\exp \left(-\beta h_{i l}\right)} & (\text { if the } i \text { th node is } \\ & \text { adjacent to the } l \text { th node) } \\ 0 & \text { (otherwise) }\end{cases}
$$

A new variable $h_{i l}$ is then introduced to express the internal state of the $i$ th neuron. Moreover, when the $i$ th and $l$ th nodes are adjacent, the internal state of the $i l$ th neuron $h_{i l}$ changes according to the following differential equation:

$$
\begin{aligned}
\frac{\mathrm{d}}{\mathrm{d} t} h_{i l}(t) & =-\sum_{k=1}^{N_{i}} J_{i k, i l} v_{i k}(t)+\eta\left\{1-\frac{1}{b_{l}}\left(q_{l}+\sum_{j \neq i}^{N_{l}} v_{j l}(t)\right)\right\} \\
& +(1-\eta)\left(1-\frac{d_{l}}{d_{c}}\right)-2 \xi\left(\sum_{k=1}^{N_{i}} v_{i k}(t)-1\right) .
\end{aligned}
$$

Further, Eq. (3) is obtained by partially differentiating Eq. (1) with respect to $v_{i l}$. In other words, Eq. (3) is equivalent to $\frac{\partial E}{\partial v_{i l}}$.

Then, from Eq. (3), we obtain,

$$
\frac{\mathrm{d} v_{i l}}{\mathrm{~d} h_{i l}}=\frac{\beta}{4 \cosh ^{2}\left(\frac{1}{2} \beta h_{i l}\right)}
$$

Thus,

$$
\frac{\mathrm{d}}{\mathrm{d} t} E=\sum_{i, l=1}^{N} \frac{\partial E}{\partial v_{i l}} \frac{\mathrm{d} v_{i l}}{\mathrm{~d} h_{i l}} \frac{\mathrm{d} h_{i l}(t)}{\mathrm{d} t}=-\sum_{i, l=1}^{N}\left(\frac{\partial E}{\partial v_{i l}}\right)^{2} \frac{\beta}{4 \cosh ^{2}\left(\frac{1}{2} \beta h_{i l}\right)} \leq 0 .
$$

Equation (5) indicates that the energy function $E$ always decreases when $h_{i l}$ changes according to Eq. (3). In other words, the energy function of Eq. (1) decreases according to the steepest descent method.

In this method, the firing of the neuron is determined using the threshold $\theta$ for the state of the neuron. The $i l$ th neuron fires if $v_{i l} \geq \theta$ and does not if $v_{i l}<\theta$. When the $i l$ th neuron fires, a packet at the buffer head of the $i$ th node is transmitted to the $l$ th adjacent node.

We now evaluate the effectiveness of the routing strategy based on the mutually connected neural networks [9] for regular and irregular topological communication networks (see Figs. 2 and 3). We conducted numerical packet routing experiments using the following procedure. First, we randomly generated packets at all nodes in the communication network according to a uniform distribution. Each packet had a randomly assigned destination. Link selection was then conducted simultaneously at every node and the packets were transmitted to the adjacent nodes. The packets transmitted from the nodes were stored at the buffer tail of the adjacent node. $d_{l}$ in Eq. (3) is set to the shortest distance from the $l$ th adjacent node to the destination of packet. Thus, $d_{l}$ dynamically changes depending



Fig. 2. Regular topological communication network with 81 nodes. This communication network was originally used by Horiguchi and Ishioka [9]. 




Fig. 3. Irregular topological communication network with 81 nodes.

on the location of destination. In addition, $d_{c}$ in Eq. (3) is selected as the longest distance among any shortest paths in the communication networks. We set the buffer size of every node to 100 and limited the number of packet movements between nodes to 20. A packet was removed when the buffer of the transmitted adjacent node was full and the packet exceeded the allowable number of packet movements. Moreover, we fixed the total number of packets in the communication network. We then added a new packet whenever a packet was removed or arrived at its destination. The source and destination of the newly added packet was randomly assigned using uniformly distributed random numbers.

Experimental simulations were conducted by the discrete time as the same ways in [9]. In these simulations, we set $\beta=3.0, \eta=0.1,0.3,0.5,0.7$, and 0.9 , and $\xi=0.3$ in Eq. (3). In addition, we set the threshold for the state of neuron $\theta=0.9$. We set the number of iterations $T$ (i.e., the link selection and packet transmission simultaneously conducted at every node) to 1,000. We conducted 10 simulations for five different values of $\eta$ and averaged the results.

To evaluate the performance of the conventional neural-based routing strategy, we used the following quantities: average number of packets at each node $(\bar{S})$, average number of packets lost before arriving at their destinations $(\bar{L})$, and average number of packets arriving at their destinations $(\bar{A})$. These quantities are defined by

$$
\begin{gathered}
\bar{S}=\frac{1}{N} \sum_{i=1}^{N} S_{i}, \\
\bar{L}=\frac{1}{T} \sum_{t=1}^{T} L(t), \\
\bar{A}=\frac{1}{T} \sum_{t=1}^{T} A(t),
\end{gathered}
$$

where $S_{i}$ is the number of packets at the $i$ th node, $T$ is the number of iterations, $L(t)$ is the number of lost packets at the $t$ th iteration, and $A(t)$ is the number of arriving packets at the $t$ th iteration.

Figure 4 shows the average number of arriving packets $(\bar{A})$ for regular (Fig. 4(a)) and irregular (Fig. 4(b)) topological communication networks. As shown in Fig. 4(a), when the average number of packets at each node $(\bar{S})$ is below 90 , all routing strategies except for the $\eta=0.1$ and $\eta=0.9$ cases achieve a higher packet arrival rate in regular topological communication networks. However, if the routing strategy is applied to irregular topological communication networks, the average number of arriving packets drastically decreases in almost all cases (Fig. 4(b)).

Figure 5 shows the average number of lost packets $(\bar{L})$ for regular (Fig. $5(\mathrm{a})$ ) and irregular (Fig. $5(\mathrm{~b})$ ) topological communication networks. In Fig. 5, although almost all the packets are transmitted to their destinations in the regular topological communication network (Fig. 5(a)), the average number of lost packets in all the $\eta$ cases increases for the irregular topological communication network (Fig. 5(b)). These results indicate that the performance of the conventional neural-based routing strategy declines when it applied to irregular topological communication networks. 

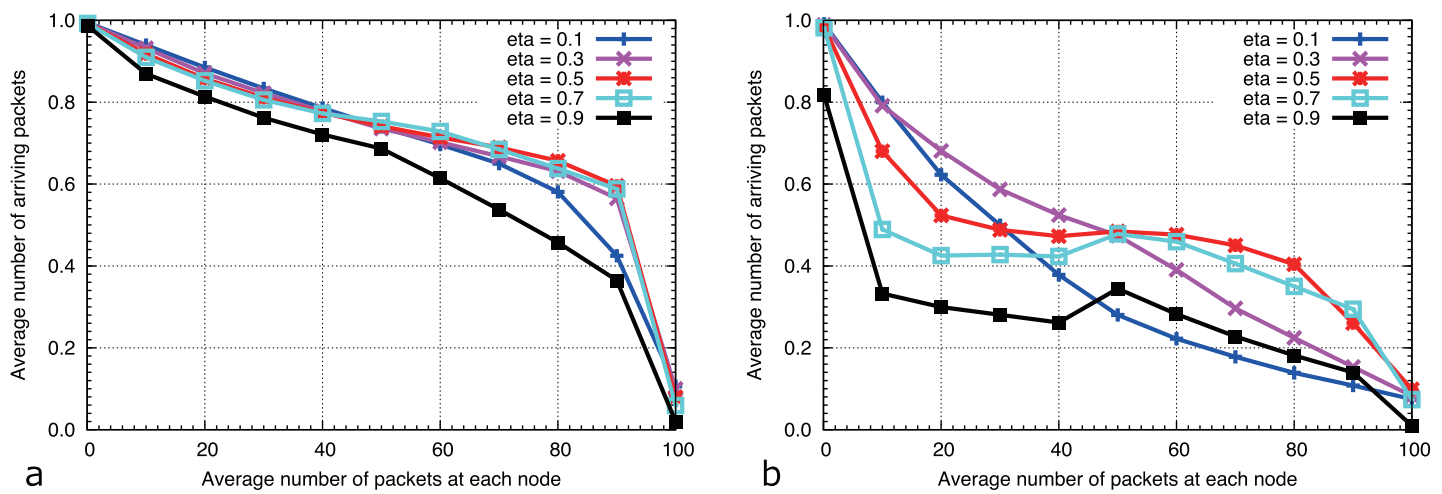

Fig. 4. Relationship between the average number of packets at each node $(\bar{S})$ and average number of packets arriving at their destinations $(\bar{A})$ for the (a) regular topological communication network (Fig. 2) and (b) irregular topological communication network (Fig. 3).
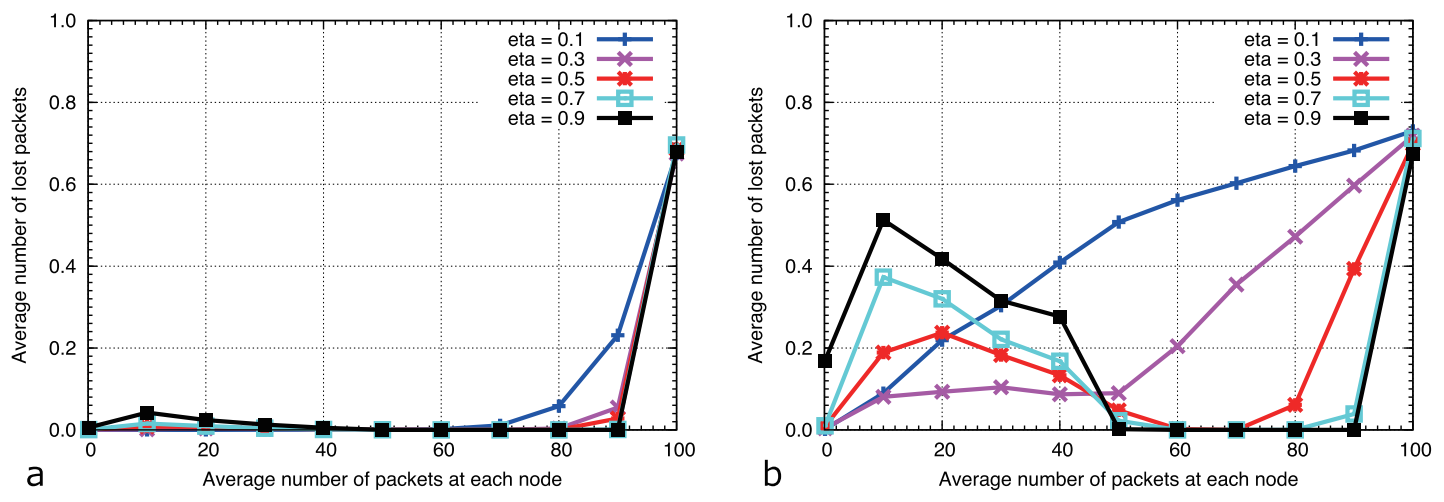

Fig. 5. Relationship between the average number of packets at each node $(\bar{S})$ and, an average number of lost packets $(\bar{L})$ for the (a) regular topological communication network (Fig. 2) and (b) irregular topological communication network (Fig. 3).

We can see that the average number of arriving packets $(\bar{A})$ for all the $\eta$ cases rapidly decreases when the average number of packets at each node $(\bar{S})$ becomes large in irregular topological communication networks. To probe further, we then measure the congestion levels of the nodes for the regular and irregular topological communication networks. The congestion level of the $i$ th node can be found using $q_{i}(t) / b_{i}\left(0<q_{i}(t) / b_{i}<1\right)$. If $q_{i}(t) / b_{i}$ evaluates to 1 , no adjacent nodes can transmit the packets to the $i$ th node at the $t$ th iteration because the $i$ th node has a full buffer. The congestion levels of the nodes using the conventional neural-based routing strategy with $\eta=0.5$ are shown in Fig. 6 . Here, we see that although the congestion levels of the nodes show similar values for the regular topological communication network (Fig. 6(a)), several nodes show higher congestion values if the strategy is applied to the irregular topological communication network, as shown in Fig. 6(b).

\section{Neural-based routing strategy with the transmission information}

In the previous section, we clarified that the conventional neural-based routing strategy [9] performs well in regular topological communication networks. However, its performance declines in the irregular topological communication network. The conventional neural-based routing strategy [9] determines the optimum adjacent node using the distance information of packets and the queuing information of adjacent nodes. Thus, even if a packet is transmitted to an adjacent node by avoiding the packet congestion among these adjacent nodes, the packet could be trapped in the congested node in the next transmission because it uses only the queuing information of adjacent nodes. To improve this situation, one possible way is to use the queuing information of all nodes in the communication networks. However, the heavy processing cost to obtain the queuing information is necessary because the queuing information always changes depending on the distribution of packets. From this viewpoint, 

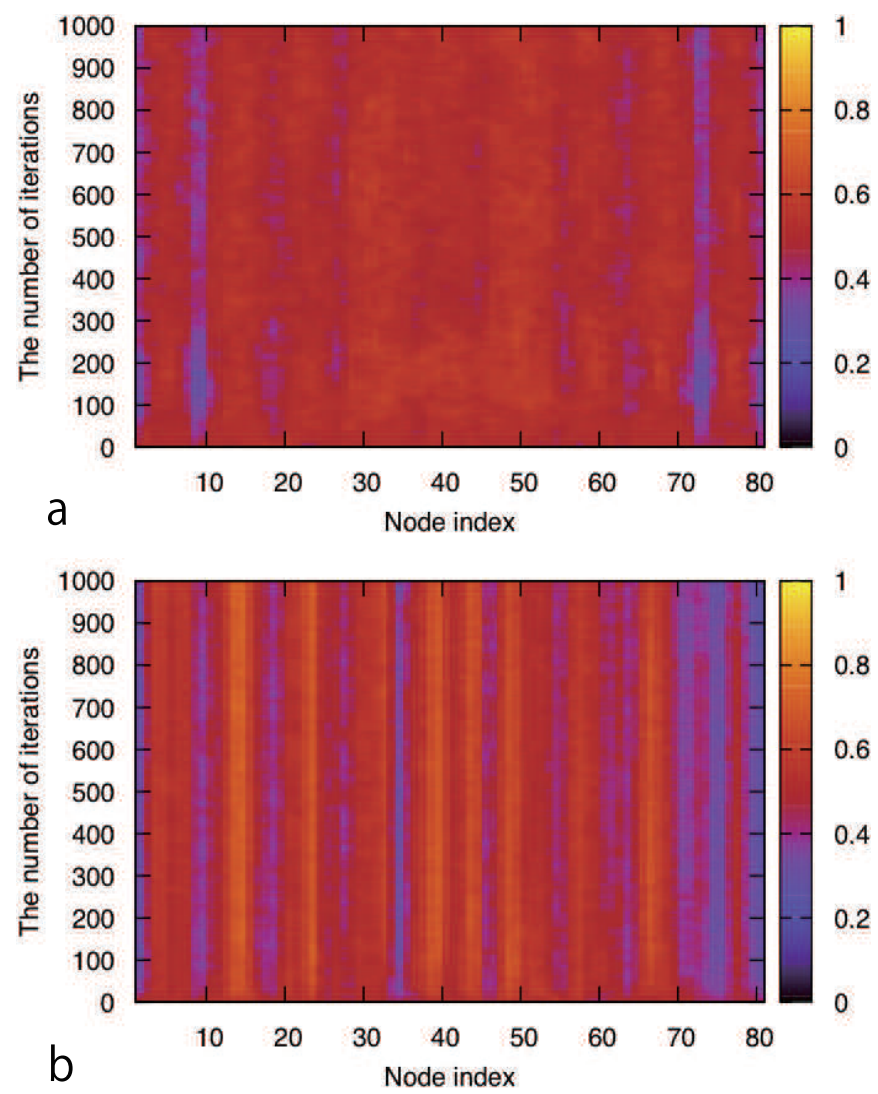

Fig. 6. Congestion levels of the nodes as a function of the number of iterations $(T)$ and node index using the conventional neural-based routing strategy with $\eta=0.5$ for the (a) regular topological network (Fig. 2) and (b) irregular topological network (Fig. 3). In these simulations, we set the average number of the packets at each node $(\bar{S})$ to 50 .

we then propose a neural-based routing strategy with transmission information along the shortest path to the destination of packet. By using this transmission information effectively, we expect that the packets are quickly transmitted to the destinations by avoiding the congested node. Here, the transmission information is the accumulated number of packets in the buffer of each node on the path to the destination.

The calculation cost of the conventional neural-based routing strategy [9] becomes $O\left(N^{3}\right)$ because it measures the packet transmission information between the $j$ th and $l$ th adjacent nodes expressed by $v_{j l}$ in the first term in Eq. (1). On the other hand, because the proposed routing strategy measures the packet transmission information along the shortest paths of the packets without using $v_{j l}$, the calculation cost becomes $O\left(\alpha N^{2}\right)=O\left(N^{2}\right)$. The proposed routing strategy then evaluates the optimum adjacent nodes much faster than the conventional neural-based routing strategy.

We implement two different types of transmission information in this paper. The first is the moving average transmission information, and the second is the exponentially averaged transmission information. This transmission information is then added to the energy function defined by Eq. (1).

\subsection{Moving average transmission information}

This strategy determines the optimal adjacent node for routing the packets by minimizing the following energy function:

$$
E_{\text {mov }}=-\eta \sum_{i=1}^{N} \sum_{l=1}^{N_{i}}\left\{1-\left(\frac{1}{\alpha}\right) \sum_{d \in R}^{\alpha}\left(\frac{q_{d}}{b_{d}}\right)\right\} v_{i l}-(1-\eta) \sum_{i=1}^{N} \sum_{l=1}^{N_{i}}\left(1-\frac{d_{l}}{d_{c}}\right) v_{i l}+\xi \sum_{i=1}^{N}\left(\sum_{l=1}^{N_{i}} v_{i l}-1\right)^{2} .
$$

In Eq. (6), $b_{d}$ is the buffer size of the $d$ th node, $q_{d}$ is the number of packets in the buffer of the $d$ th node, $R$ is the set of nodes on the shortest path from the $i$ th node to the destination of the packet, 
and $\alpha$ is the control parameter that determines the acquisition range of the transmission information from the $i$ th node. The difference between the original neural-based routing strategy of Eq. (1) and this routing strategy is that the moving average transmission information is introduced in the first term, namely, $(1 / \alpha) \sum_{d \in R}^{\alpha}\left(q_{d} / b_{d}\right)$.

\subsection{Exponentially averaged transmission information}

This neural-based routing strategy evaluates the optimal adjacent node for routing packets using exponentially averaged transmission information. In this case, the energy function is defined as follows:

$$
E_{\text {exp }}=-\eta \sum_{i=1}^{N} \sum_{l=1}^{N_{i}}\left\{1-\left(\frac{\sum_{d=1}^{\alpha}\left(k^{d-1} \frac{q_{d}}{b_{d}}\right)}{\sum_{d=1}^{\alpha} k^{d-1}}\right) v_{i l}\right\}-(1-\eta) \sum_{i=1}^{N} \sum_{l=1}^{N_{i}}\left(1-\frac{d_{l}}{d_{c}}\right) v_{i l}+\xi \sum_{i=1}^{N}\left(\sum_{l=1}^{N_{i}} v_{i l}-1\right)^{2} .
$$

In Eq. (7), $k(0<k<1)$ is the decay parameter of the transmission information. In the same manner as in the moving average transmission information, this routing strategy exponentially averages the transmission information using the first term in Eq. (7).

In both these neural-based routing strategies, the congested nodes will be effectively avoided by introducing the transmission information of the packets to the destinations. The packets will then be reliably transmitted to their destinations.

\section{Numerical experiment}

In this section, we evaluate the performance of four routing strategies. The first is the proposed neural-based routing strategy with the moving average transmission information and the second is the same strategy with the exponentially averaged transmission information. The third is the originally proposed neural-based routing strategy [9], and the last is the shortest path strategy that is commonly employed in real-world systems. We applied these routing strategies to the irregular topological communication network (Fig. 3) and scale-free networks [12].

In these simulations, we adopted the same experimental assumptions and parameter settings as those in Section 2. We fixed $\alpha=2.0, k=0.6$, and $\eta=0.5$ in Eqs. (6) and (7) in these simulations.

First, we evaluated the performance of the two types of proposed neural-based routing strategies as mentioned in the previous section, the original neural-based routing strategy [9], and the shortest path routing strategy in the irregular topological communication network (Fig. 3). We evaluated these strategies using the average number of packets arriving at their destinations $(\bar{A})$ and the average number of packets lost before arriving at their destinations $(\bar{L})$.
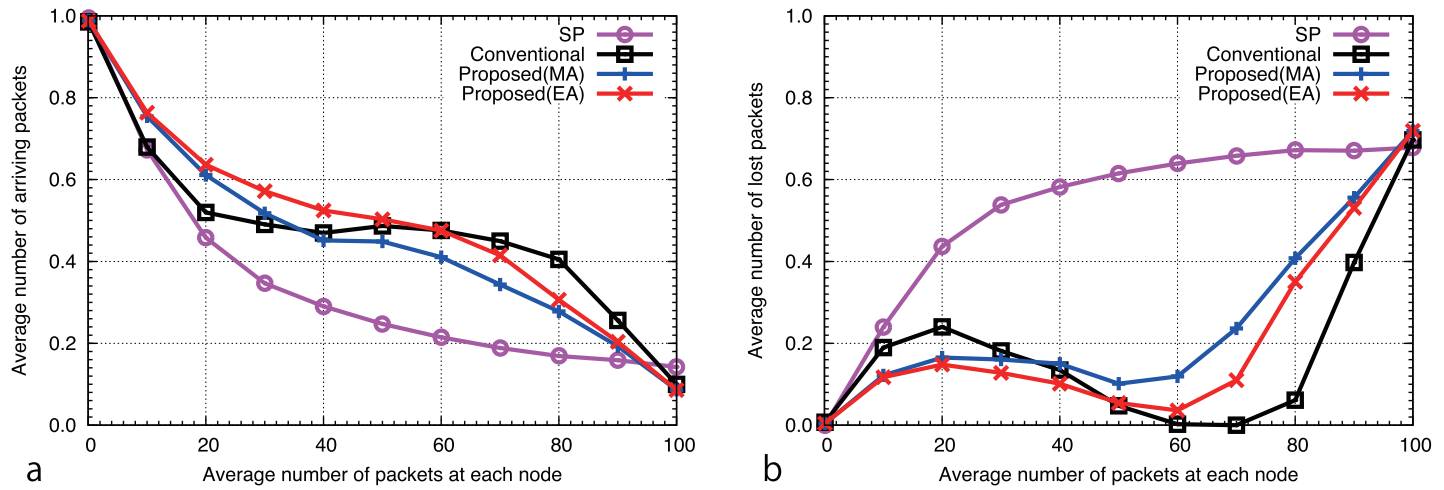

Fig. 7. Relationship of the average number of packets at each node $(\bar{S})$ to the (a) average number of packets arriving at their destinations $(\bar{A})$, and (b) average number of packets lost before arriving at their destinations $(\bar{L})$ for the irregular topological communication network (Fig. 3).

Figure 7 shows the average number of packets arriving at their destinations $(\bar{A})$, and the average number of packets lost before arriving at their destinations $(\bar{L})$ for the irregular topological communication network (Fig. 3). In Fig. 7(a), the proposed neural-based routing strategy with the 
exponentially averaged transmission information (EA) achieves a better arrival rate than the proposed routing strategy with the moving average transmission information (MA), the conventional neuralbased routing strategy (Conventional) and the shortest path approach (SP) when the average number of packets at each node $(\bar{S})$ is below 50 . However, the Conventional strategy shows higher arrival rate than the MA, EA, and SP strategies if the $\bar{S}$ is larger than 60. In addition, as shown in Fig. 7(b), the EA strategy loses fewer packets than the other routing strategies when the average number of packets at each node is less than 50 .

From these results, we can see that the performance of the conventional neural-based routing strategy is enhanced by adding the transmission information. In particular, EA plays a significant role in alleviating packet congestion if a small number of packets are flowing in the communication networks.

In particular, because existing communication networks exhibit the scale-free property [13], it is of interest to consider scale-free networks [12]. In the same manner as described in the previous simulations, we evaluated the performance of the two types of the proposed neural-based routing strategies, the Conventional and SP strategies in the scale-free network.

Scale-free networks are constructed by the following procedure [12]. We begin with a complete graph of four nodes. We then insert a new node with four links at every time step. Next, we connect the four links of the newly added node to the nodes that already exist in the network with probability $\Pi\left(k_{i}\right)=k_{i} / \sum_{j=1}^{N} k_{j}$, where $k_{i}$ is the degree of the $i$ th node $(i=1, \ldots, N)$, and $N$ is the number of the nodes at the current iteration.


Fig. 8. Relationship of the average number of packets at each node $(\bar{S})$ to the (a) average number of packets arriving at their destinations $(\bar{A})$, and (b) average number of packets lost before arriving at their destinations $(\bar{L})$ for the scale-free network.

Figure 8 shows the average number of packets arriving at their destinations $(\bar{A})$ and the average number of packets lost before arriving at their destinations $(\bar{L})$ for the scale-free networks. In Fig. 8(a), the EA strategy achieves a better arrival rate than the other routing strategies when the average number of packets at each node $(\bar{S})$ is less than 70 . In addition, in Fig. 8(b), the EA loses fewer packets than the other routing strategies.

The node congestion levels for the Conventional and EA strategies for the scale-free networks are shown in Fig. 9. Here, we see that although both the Conventional and EA strategies show similar congestion levels for scale-free networks, some nodes using the EA strategy show lower congestion levels than those using the Conventional strategy.

To implement the routing strategy into real-world systems, it is necessary to check the robustness of proposed routing strategy for various parameter settings. We then evaluate the robustness of the EA strategy by the irregular topological communication network (Fig. 3) and scale-free networks. Because vital parameters that determines the performance of EA strategy defined by Eq. (7) are $\eta$ that decides the priorities of the first and second terms, and $\alpha$ that determines the acquisition range of the transmission information, we measured the average number of packets arriving at their destinations $(\bar{A})$ for various $\eta$ and $\alpha$ cases. 



Fig. 9. Congestion levels of the nodes as a function of the number of iterations $(T)$ and the node index by the (a) conventional neural-based routing strategy with $\eta=0.3$ and (b) proposed neural-based routing strategy with the exponentially averaged transmission information for the scale-free networks. In these simulations, we set the average number of packets at each node $(\bar{S})$ to 40 .

The average number of packets arriving at their destinations $(\bar{A})$ as a function of parameters $\eta$ and $\alpha$ in Eq. (7) by the irregular topological communication network (Fig. 3) and the scale-free networks are shown in Fig. 10. Here, we see that if $\eta$ is in between 0.2 and 0.4 and $\alpha$ is in between 2 and 3 (Fig. 10(a)), the EA strategy achieves a better arrival rate of the packets in the irregular topological communication network. In addition, the EA strategies shows good performance if $\eta$ is in between 0.4 and 0.7, and $\alpha$ is in between 2 and 3 for the scale-free networks (Fig. 10(b)).

From these results, we can see that the exponentially averaged transmission information is the key to quickly transmit packets in scale-free networks. As we have already mentioned, real packet communication networks have a scale-free property, and hence these results indicate that the neural-based routing strategy with the transmission information has potential as a strategy to alleviate the packet congestion that frequently occurs in real packet communication networks.

\section{Conclusion}

In this paper, we proposed two types of neural-based routing strategies with the transmission information, namely, one with moving average transmission information and another with the exponentially averaged transmission information. These routing strategies determine the optimal adjacent node for transmitting the packet using the distance and the transmission information (the accumulated number of the packets on the paths to the destinations). We then evaluated the routing strategies in irregular topological communication networks and scale-free networks. From the numerical experiments, we confirmed that the performance of the neural-based routing strategy with exponentially averaged transmission information performs better than the originally proposed routing strategy and 

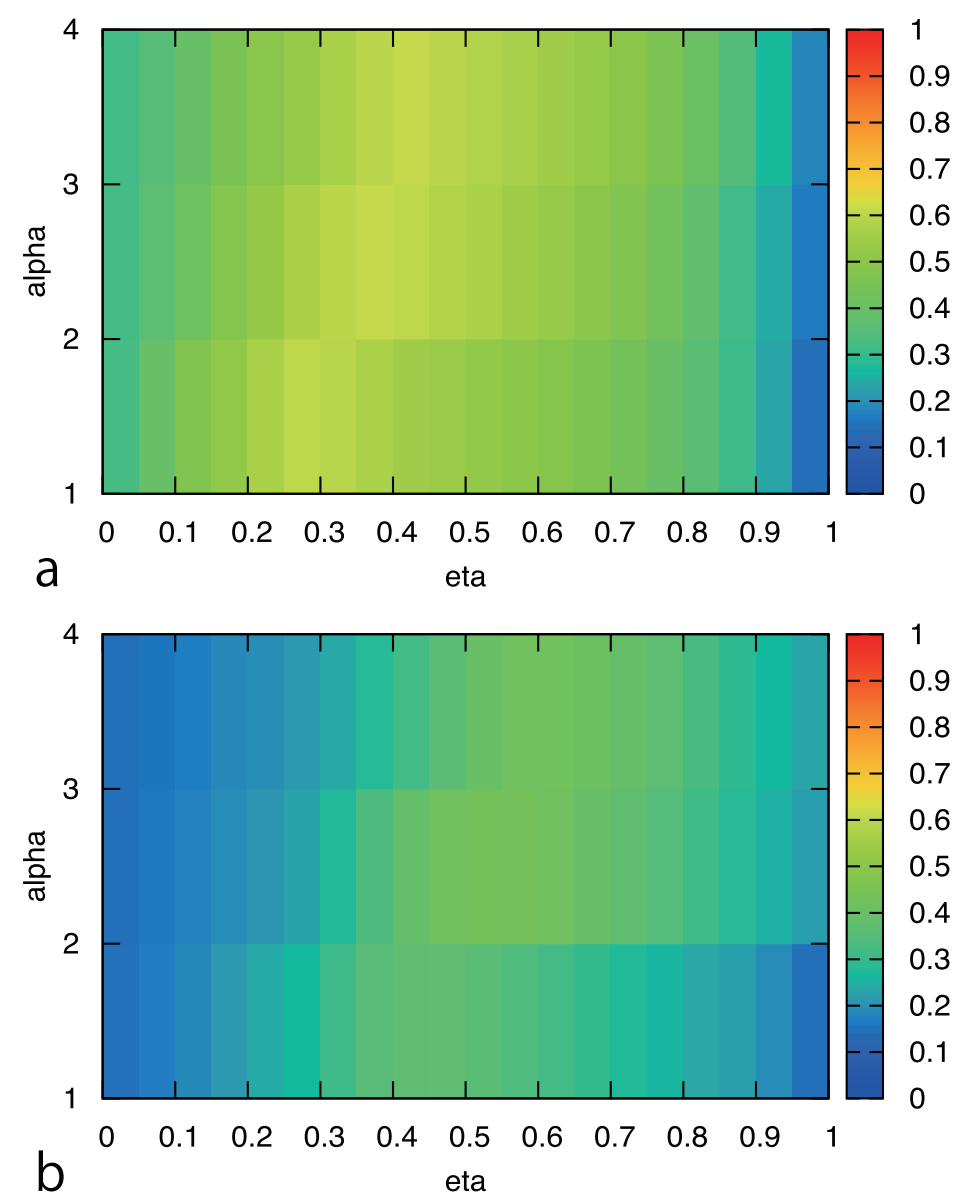

Fig. 10. Average number of packets arriving at their destinations $(\bar{A})$ by the proposed EA strategy as a function of $\eta$ and $\alpha$ in Eq. (7) by the (a) irregular topological communication network (Fig. 3) and (b) scale-free networks. In these simulations, we set the average number of packets at each node $(\bar{S})$ to 30 .

the shortest path approach that is commonly employed by real packet communication networks. However, the proposed routing strategies and the conventional load-distribution strategy show almost the same performance if a large number of packet flows in communication networks. To improve this, we will then analyze the declines of the proposed strategy by this case in the future work. Further, in this paper, we assumed that the information of the accumulated number of packets on the path was instantaneously obtained by every node. In future, we will consider how to deal with the delay of this information in order to implement the proposed neural-based routing strategy into real-world systems. In addition, we plan to evaluate the implementability of our proposed neural-based routing strategy using a network simulator.

\section{References}

[1] T. Ohira and R. Sawatari, "Phase transition in a computer network traffic model," Physical Review E, vol. 58, no. 1, pp. 193-195, 1998.

[2] E.W. Dijkstra, "A note on two problems in connexion with graphs," Numerische Mathematik, vol. 1, pp. 269-271, 1959.

[3] R.E. Bellman, "On a routing problem," Ouarterly of Applied Mathematics, vol. 16, pp. 87-90, 1958.

[4] S.E. Dreyfus, "An appraisal of some shortest-path algorithms," Operations Research, vol. 17, pp. 395-412, 1969.

[5] R.W. Floyd, "Algorithms 97: Shortest path," Communications of the ACM, vol. 5, p. 345, 1962.

[6] P. Echenique, J. Gómez-Gardenes, and Y. Moreno, "Improved routing strategies for internet 
traffic delivery," Physical Review E, vol. 70, p. 056105, 2004.

[7] A. Arenas, A. Díaz-Guilera, and R. Guimerà, "Communication in networks with hierarchical branching," Physical Review Letters, vol. 86, no. 14, pp. 3196-3199, 2001.

[8] L. Zhao, Y.-C. Lai, K. Park, and N. Ye, "Onset of traffic congestion in complex network," Physical Review E, vol. 71, p. 026125, 2005.

[9] T. Horiguchi and S. Ishioka, "Routing control of packet flow using a neural network," Physica A, vol. 297, pp. 521-531, 2001.

[10] T. Kimura, H. Nakajima, and T. Ikeguchi, "A packet routing method for complex networks by a stochastic neural network," Physica A, vol. 376, pp. 658-672, 2007.

[11] J.J. Hopfield and D.W. Tank, "Neural computation of decisions in optimization problems," Biological Cybernetics, vol. 52, pp. 141-152, 1985.

[12] A.L. Barabási and R. Albert, "Emergence of scaling in random networks," Science, vol. 286, pp. 509-512, 1999.

[13] M. Faloutsos, F. Faloutsos, and C. Faloutsos, "On power-law relationships of the internet topology," Comput. Coomun. Rev., vol. 29, pp. 251-262, 1999. 